\title{
Non-simultaneous two-stage detection of spontaneous bilateral isthmic tubal pregnancy
}

\author{
Agnieszka Gardyszewska ${ }^{1}$, Agnieszka Dobrowolska-Redo ${ }^{1}, K^{\prime}$ rzysztof Czajkowski ${ }^{1}$, \\ Roman Smolarczyk ${ }^{2}$, Piotr Roszkowski ${ }^{1}$ \\ ${ }^{1} 2^{\text {nd }}$ Department of Obstetrics and Gynecology, Warsaw Medical University, Poland \\ ${ }^{2}$ Department of Gnecological Endocrinology, Warsaw Medical University, Poland
}

\section{INTRODUCTION}

The incidence of ectopic pregnancies ranges between 1.5 and $2 \%$ of all pregnancies. The surmised risk factors for ectopic implantation include advanced maternal age, endometriosis, a history of adnexitis, a history of tubal pregnancies and tubal surgeries, pelvic adhesions, and congenital defects of the uterus. Bilateral tubal pregnancies are very rare. The incidence of these pregnancies ranges between $1 / 1.580$ and $1 / 725$ of all ectopic pregnancies.

\section{CASE REPORT}

The patient, aged 27, was admitted to our hospital in December 2011 due to suspected ectopic pregnancy and genital spotting persisting for 9 days. The patient reported no history of chronic diseases or surgeries. The pregnancy was her first. She had never used contraception, could not remember the date of her last menstrual period, and had had no sexual intercourse for 3 weeks.

A transvaginal ultrasound scan revealed a structure with a diameter of $18 \mathrm{~mm}$ located next to the left ovary. The $\beta$-hCG level was $1.219 \mathrm{mIU} / \mathrm{mL}$. The patient was qualified for a laparoscopic procedure. The surgery revealed a retroverted uterine trunk of normal size and ovaries and a right fallopian tube of normal shape and condition. The isthmus of the left fallopian tube was dilated up to $2 \mathrm{~cm}$ along a section of $2 \mathrm{~cm}$ and was cyanosed. Tubal milking with tissue removal was performed. A histopathological examination confirmed the presence of chorionic villi in the collected material.

The follow-up $\beta$-hCG level, as measured 10 days after the surgery, was $3.300 \mathrm{mlU} / \mathrm{mL}$. The patient did not agree to be hospitalized.

The patient reported to the hospital 21 days after the laparoscopy. A transvaginal ultrasound scan revealed a lesion with a diameter of $37 \mathrm{~mm}$ located in the vicinity of the right adnexa. The surgery revealed dilation of the isthmus of the right fallopian tube that was $25 \mathrm{~mm}$ in diameter and $40 \mathrm{~mm}$ in length. A histopathological examination revealed the presence of a trophoblast and chorionic villi within the tube.

\section{DISCUSSION}

There are many theories regarding the development of such pregnancies. One theory includes polyovulation, with fertilized oocytes undergoing pathological implantation in both fallopian tubes. Another possibility may involve migration of trophoblast cells from one tube into the another. Another potential theory involves ovulation in the initial stages of pregnancy.

In our case, the pregnancy within the second tube could not be observed during the first surgery. Despite the fact that laparoscopy appears to be the best imaging technique for ectopic pregnancies, the rate of undetected pregnancies ranges between 3 and $4 \%$.

The symptoms of bilateral tubal pregnancy are similar to the symptoms of unilateral tubal pregnancy. The diagnosis is most often made during surgery. In case of our patient, the second ectopic pregnancy was not manifested during the early postoperative period. The suspicion of persistent ectopic pregnancy was proposed on the basis of $\beta$-hCG level monitoring. According to the available literature, the interval between the diagnoses of the first and second ectopic pregnancies ranges from several days to several weeks. 